\title{
Arbeitsorientierung und Digitalisierung: Menschengerechte, menschenwürdige Gestaltung guter Arbeit, national und international
}

\author{
Manfred Schweres ${ }^{1}$
}

๑) Springer-Verlag GmbH Deutschland, ein Teil von Springer Nature 2018

Dieses Heft steht in der Kontinuität ähnlich ausgerichteter Arbeiten (Schweres 2008 bis Schweres und Breisig 2015; Schweres und Lippoth 2013). Viele Z.Arb.wiss.-Beiträge bewegen sich eher auf den unteren Ebenen der Systematik des arbeitswissenschaftlichen Gegenstandskatalogs. Arbeitsorientierungsbeiträge liegen häufig in den Ebenen 6. Betriebliche Arbeitsbeziehungen und Organisation sowie in 7. Arbeit und Gesellschaft (Luczak und Volpert 1987, S. 63).

„Globalisierung und Digitalisierung der Wertschöpfung führen unter dem Aspekt nachhaltiger und damit auch humaner Arbeitssysteme zu neuen Herausforderungen für die Arbeitswissenschaft. ... [Das betrifft] die Betrachtung organisationsübergreifender, vollständiger Wertschöpfungsketten, die meist international sind" (Zink 2017, S. 92). Damit ergeben sich - national und international - für die Beschäftigten häufig inhumane, unsoziale, nicht ökologische Arbeits-/Entgeltbedingungen. Die Arbeitsforschenden werden damit in ihrem Berufsethos angesprochen: „Wissenschaft - das heißt auch: gesellschaftspolitische Verantwortung!“ (Hey 2018).

Für Theoriediskussion und Gestaltungspraxis ist hilfreich, was K. J. Zink einleitend über die Zukunft humaner Gestaltung der Arbeit in einer digitalisierten Welt vorstellt. Er betrachtet (1) die Ergebnisse des Dialogprozesses des Bundesministeriums für Arbeit und Soziales (BMAS) zum Weißbuch Arbeiten 4.0 und (2) die Vorschläge der Kommission „Arbeit der Zukunft“ der Hans-Böckler-Stiftung sowie (3) Ergebnisse der von der Internationalen Arbeitsorganisation (ILO) eingesetzten „Global Commission on the Future of Work“. In der zusammenfassenden Bewertung zieht er das GfA-Memorandum „Die Zukunft der Arbeit erforschen“ (GfA 2000) heran. Danach ,,..., ist es doch sehr erstaunlich, wie viele der von der Kommission ,Arbeit der

Prof. Manfred Schweres

manfred.schweres@iadm.info

1 Arbeitswissenschaft und Didaktik des Maschinenbaus, LUH, Hannover, Deutschland
Zukunft' thematisierten Problemfelder dort schon zu finden sind".

Die Arbeitswissenschaft ,,... besteht aus einem naturwissenschaftlichen und einem sozialwissenschaftlichen Teil“" (Preuschen 1973, S. 7). Dem Beitrag der Soziologie und der Sozialforschung im Verhältnis zu einer interdisziplinären Arbeitswissenschaft widmet sich I. Raehlmann (u.a. Raehlmann 2011; Raehlmann und Volpert 1997). Von den Ursprüngen der Soziologie und der Arbeitswissenschaft schlägt sie den historischen Bogen hin zur HdA-Forschung. Neu ist die Aufbereitung eines unveröffentlichten Vortrages von O. Neuloh auf der GfA Herbstkonferenz 1972 in Linz (Donau). Die Auseinandersetzungen um die Deutungshoheit in der Arbeitsforschung, I. Raehlmann verweist darauf erneut, sind allen Arbeitsforschenden bekannt.

Was bedeuten heute Arbeitsorientierung(en) in betriebswirtschaftlicher/personalwirtschaftlicher Perspektive, vor allem in erweiterten Mensch-Maschine-ArbeitsumweltSystemen (Netzwerkorganisationen; Wertschöpfungsketten)? So lautete die Anfrage an St. Laske: Vorarbeiten (Laske und Schweres 2014) und weiterführende Überlegungen (Zink 2014 - vgl. Picot et al. 2013; Sydow und Helfen 2015; Ulich 1996). In Blitzlichtern verdeutlicht der Autor einleitend die negativen Veränderungen in Arbeitsverhältnissen und Arbeitsbedingungen für eine immer größere Zahl abhängig Beschäftigter. Wie weit stellt sich arbeitsbezogene Wissenschaft auch ethisch-normativ den neuen Fragestellungen? Ob diese aber aufgegriffen werden, ist danach ,... von drei zentralen Faktorenbündeln abhängig: (1) ... forschungsleitendes Interesse und Problemwahrnehmung; ... (2) Nutzen der Bearbeitung entsprechender Themen für wissenschaftliche Reputation und Karrieremöglichkeiten ...; (3) Zugang zu Forschungsmitteln“. Er konzentriert sich auf das erste Faktorenbündel.

Die folgende Synopse von Arbeitsorientierung(en) erörtert er unter (1) „Beforschung von arbeitsrelevanten Themen", stellt dagegen (2) die Gruppe von Fachkollegen, die von einem normativ kritischen Konzept ausgehen. Schließlich betrachtet er (3) die Chancen eines interdisziplinär orientierten Zugangs zu „Arbeitsorientierung“. Ausgehend 
von den positiven Entwicklungen von Arbeitsbedingungen und Arbeitsforschung in der Reformphase (1960er/1970er Jahre) untersucht er die Auswirkungen der „Vermarktlichung" in den 1990er Jahren auf Arbeitsorientierung. Abschließend erweitert er seine Betrachtungen hin auf das Aufbrechen (nationaler) Unternehmensstrukturen zu ,grenzenlosen Unternehmungen“ "mit neuen Formen der Produktions- und Arbeitsorganisation und deren destruktive Folgen für die Beschäftigten (siehe „Blitzlichter“). Zusammenfassend leitet er aus der Synopse weiterführende Frage-/ Themenstellungen an Arbeitsforschung und deren Umsetzung in eine menschengerechte Gestaltung Guter Arbeit ab. ,... ein Schritt zur Revitalisierung früherer Ansätze zur Humanisierung der Arbeit. Dringlich wäre dies".

"Arbeitsorientierung in den Wirtschaftswissenschaften" (Laske und Schweres 2014) war eine Bestandsaufnahme, die u. a. die Themenfelder „Arbeit“ (Breisig und Schweres 2015; Breisig 2014a) und „Arbeitsproduktivität“ (Breisig 2014b; Weiß 2011) ansprach. So setzt sich Th. Breisig mit dem umstrittenen Begriffsfeld „Leistung“ auseinander. Steigende Leistungsanforderungen an Beschäftigte sind verbunden mit einer Vorstellungsverschiebung, was deren „Leistung“ ausmacht: (1) Sind es die menschlichen Leistungsvoraussetzungen (Leistungsfähigkeit/-bereitschaft; Schmidtke 1981), welche die Arbeitsperson in die Bewältigung einer Arbeitsaufgabe einbringt? (2) Oder zählt allein das Leistungsergebnis (betriebswirtschaftliche Erfolgskriterien)? Der Autor belegt das vorherrschende Verständnis der Beschäftigten, wonach ,... das Leistungsprinzip und eine Vorstellung von differenzierender Leistungsgerechtigkeit zum festen Kern der Orientierungen gehört" (vgl. Tullius und Wolf 2016). Das sollte bei der Arbeitssystemgestaltung nach den bekannten arbeitswissenschaftlichen Bewertungs-/Beurteilungskriterien (u.a. Luczak und Volpert 1987) arbeitsbezogenes Fachpersonal und die Interessenvertretungen anleiten.

Arbeitsproduktivität in der betrieblichen Leistungserstellung bestimmt den Beitrag von T. Jeske und Mitautoren. Die arbeitsorganisatorischen/betriebswissenschaftlichen Praxisbeispiele aus der Nutzung der Digitalisierung für das Produktivitätsmanagement wurden nach Schwerpunkten quantitativer Veränderungen an der betrieblichen Leistungserbringung (Input/Output) geordnet, vor allem nach informatorisch dominierten Arbeitsformen. „Diese Schwerpunkte werden entlang des Datenhandhabungsprozess analysiert und die dabei abgedeckten Einsatz- bzw. Anwendungsspektren aufgezeigt. Zudem werden schwach besetzte Bereiche des Ordnungs- und Gestaltungsrahmens aufgezeigt und diskutiert". In folgenden Untersuchungen sollen vor allem kleine und mittlere Betriebe mit verbesserten Ordnungs-/ Gestaltungsstrukturen bei der Bewältigung der Digitalisierungsschübe unterstützt werden.
Der Teil-Schwerpunkt „Fleischindustrie/Fleischwirtschaft" ist inhaltlich eng mit den vorangehenden Beiträgen verknüpft. Zum erleichterten Verständnis für den Leserkreis sind am Ende des Heftes die „Beweggründe“ des Editors abgedruckt.

„Menschenwürdige Arbeit“ war der aufrüttelnde Text von Pfarrer Peter Kossen (Kossen 2017) überschrieben. Er gab letztlich den Anstoß für diesen ungewöhnlichen Teilschwerpunkt und für den Praxisbericht (Basisartikel) von P. Kossen. Neue Geschäftsmodelle auf nationaler und internationaler Ebene verschlechtern die Arbeitsbedingungen der Beschäftigten in der Fleischindustrie und rufen klärenden Forschungsbedarf hervor (siehe Beweggründe).

Die sozialethische Betrachtung von A. Küppers und U. Nothelle-Wildfeuer setzt aus katholischer Sicht an den Strukturumbrüchen in der Fleischindustrie an (vgl. C. Weinkopf). Diese führten zu erheblichen Fehlentwicklungen in den Arbeitsverhältnissen. Die Vertragssituation der Werkvertragsarbeitskräfte aus Osteuropa spiegelt die Verhandlungsungleichgewichte der Vertragspartner wider. Sie erfordern eine verbesserte soziale Arbeits- und Ordnungspolitik, eine wirkliche Soziale Marktwirtschaft.

Das neue „Gesetz zur Sicherung von Arbeitnehmerrechten in der Fleischindustrie" kann nur ein erster Schritt sein. Es bedarf aber verschärfter Behördenkontrollen. Offensichtliche Mängel in der Arbeitsgestaltung/-organisation, mangelhafte Entgeltbedingungen, zusätzlich menschenunwürdige Wohnbedingungen ergeben ein breites Forschungsund Gestaltungsfeld zur Wiedererlangung menschenwürdiger Arbeitsbedingungen in der Fleischindustrie.

A. Mayert konzentriert sich auf die europäischen Dimensionen menschenungerechter Arbeit in der Fleischindustrie, da diese bereits mit der EU-Entsenderichtlinie 1996 sich verschlechterten. Letztlich dominiert europäisches Wettbewerbsrecht (Systemwettbewerb), die „Sozialunion“ ist unterentwickelt. Das Beschäftigungskonstrukt für osteuropäische Werkvertragsarbeitnehmer in Deutschland mit gefährlicher, körperlich fordernder, schmutziger Arbeit in kleinteiligen, angeblich eigenständigen „Werken“ wird letztlich von den ausländischen Entsendebetrieben bestimmt (einschließlich Lohn-/Sozialdumping). Aber: Eine Arbeitstätigkeit, wie das hochgradig arbeitsteilige Zerlegen von Schweinen, ist kein in sich abgeschlossenes, endliches „Werk“. Der Europäische Gerichtshof hatte nur die Dienstleistungsfreiheit im Blick.

Wie schon aus katholischer Perspektive geschildert, herrschen hier weitgehend asymmetrische Machtverhältnisse am Arbeitsmarkt. Europäische Rechtsprechung erzeugt zusätzlichen sozialen Rückschritt, der nicht nur evangelischer Sozialethik widerspricht. Die tatsächlichen Arbeitsbedingungen sind voller Missstände. Mit negativen Geschäftsmodellen verschaffen sich die Riesen der Fleischindustrie 
(zusammen mit den Entsendebetrieben) zu Lasten der Beschäftigten große Konkurrenzvorteile.

Eine vertiefte Einführung in die Beschäftigtenstrukturen der Fleischwirtschaft gibt C. Weinkopf. Aus ihrer vergleichenden Darstellung der Fleischwirtschaftsstrukturen in Deutschland und in Dänemark wird deutlich, wie negativ sich die deutschen Werkvertragsverhältnisse im Verhältnis zu tarifvertraglich gesicherten dänischen Arbeitsbedingungen auswirken. Der endlich eingeführte Mindestlohn der deutschen Fleischindustrie wird weiterhin unterschritten. Das im Herbst 2016 verabschiedete „Gesetz gegen den Missbrauch von Leiharbeit und Werkverträgen“" ist nur so wirksam, wie die staatlichen Kontrollbehörden für seine Durchsetzung sorgen. Und da fehlt es nicht nur an Personal. Jetzt werden vom „Gesetz zur Sicherung von Arbeitnehmerrechten in der Fleischwirtschaft" (GSA Fleisch) mit seiner Generalunternehmerhaftung notwendige Verbesserungen erwartet. Die arbeitszeitorganisatorischen Vorgaben (u.a. Arbeitsstunden-Aufzeichnungen) erschweren das Unterlaufen von Mindestlöhnen und führen zu gesundheitsgerechteren Arbeitsstrukturen.

Letztlich wird aber nur - wie in Dänemark - der Verzicht auf Werkvertragskonstrukte und die Rückkehr in tarifvertraglich gesicherte, sozialversicherungsgeschützte Arbeitsvertragsverhältnisse solche Arbeitsbedingungen bringen, die von den Arbeitsforschenden bei ihren Untersuchungen als menschengerecht und menschenwürdig einzustufen sind.

Die gesellschaftspolitische Verantwortung der Wissenschaft hat unsere GfA eindeutig in ihrer Denkschrift verankert:

Die Gesellschaft für Arbeitswissenschaft e. V. und deren Mitglieder fühlen sich dem gesellschaftlichen Ziel verpflichtet, wo immer möglich Beiträge zur Erhaltung, Schaffung und (angemessenen) Verteilung von humaner, wirtschaftlicher und umweltverträglicher Arbeit zu leisten. (GfA 1999)

\section{Literatur}

Breisig Th (2014a) Betriebliche Leistungssteuerung - Grundlagen und Strategien für Betriebsräte. Bund-Verlag, Frankfurt/M.

Breisig Th (2014b) Perspektiven von Produktivität - Plädoyer für ein erweitertes Verständnis von Produktivität und Arbeit. In: Laske St, Schweres M (Hrsg) Arbeitsorientierung in den Wirtschaftswissenschaften. Rainer Hampp, München, Mering, S 165-186
Breisig Th, Schweres M (2015) Über 50 Jahre "Wöhe" - von Arbeit nur Spurenelemente - Das Fach im Spiegel des Lehrbuchs "Allgemeine Betriebswirtschaftslehre" von Günter Wöhe. Zent Arbeitswiss 69(4):233-238. https://doi.org/10.1007/bf03395985

GfA (1999) Selbstverständnis der Gesellschaft für Arbeitswissenschaft e. V. https://eldorado.tu-dortmund.de/bitstream/2003/28307/1/ zink.pdf

GfA (2000) Die Zukunft der Arbeit erforschen. Ein Memorandum der Gesellschaft für Arbeitswissenschaft e.V. zum Strukturwandel der Arbeit. http://www.gesellschaft-fuer-arbeitswissenschaft.de/ inhalt/dokumente/gfa-memorandum-2013.pdf

Hey J (2018) Wissenschaft - das heißt auch: gesellschaftspolitische Verantwortung. DHV-Jahresbericht, Bd. 2017. Deutscher Hochschulverband, Bonn

Kossen P (2017) Menschenwürdige Arbeit. Kirche + Leben Nr. $38 / 24.09 .2017, \mathrm{~S} 2$

Laske St, Schweres M (2014) Arbeitsorientierung in den Wirtschaftswissenschaften. Rainer Hampp, München, Mering

Luczak H, Volpert W et al (1987) Arbeitswissenschaft: Kerndefinition - Gegenstandskatalog - Forschungsgebiete. RKW, Eschborn

Picot A, Reichwald R, Wigand RT (2013) Die grenzenlose Unternehmung - Information, Organisation und Management, 5. Aufl. Gabler, Wiesbaden

Preuschen G (1973) Einführung in die Arbeitswissenschaft. Rombach, Freiburg i. Br.

Raehlmann I (2011) Interdisziplinäre Arbeitswissenschaft in der $\mathrm{Hu}$ manisierungsforschung und -politik. Ein überfälliger Neustart. Zent Arbeitswiss 65(1):9-17

Raehlmann I, Volpert W (1997) Geschichte und Richtungen der Arbeitswissenschaft. In: Luczak H, Volpert W (Hrsg) Handbuch Arbeitswissenschaft. Schäffer-Poeschel, Stuttgart, S 19-25. ISBN 978-3791007557

Schmidtke H (Hrsg) (1981) Lehrbuch der Ergonomie. Carl Hanser, München, Wien

Schweres M (2008) 35 Jahre „Menschengerechte Gestaltung der Arbeit" (MGdA). Zent Arbeitswiss 62(3):227-234

Schweres M, Breisig Th (2015) Editorial: Menschengerechte Gestaltung guter Arbeit (MGgA). Zent Arbeitswiss 69(4):194-196. https://doi.org/10.1007/bf03395977

Schweres M, Lippoth KU (2013) Humanisierung und Wirtschaftlichkeit - Für eine neue Humanisierungsinitiative. Eine Zusammenschau. Zent Arbeitswiss 67(1):41-43

Sydow J, Helfen M (2015) Arbeit in Netzwerkorganisationen. Zent Arbeitswiss 69(4):223-226

Tullius K, Wolf H (2016) Moderne Arbeitsmoral: Gerechtigkeitsund Rationalitätsansprüche von Erwerbstätigen heute. Wsi Mitt 69(7):493-502

Ulich E (1996) Neue Produktionskonzepte - ein kritischer Diskussionsbeitrag. Zent Arbeitswiss 50(4):193-198

Weiß Th (2011) Arbeitsproduktivität, nicht nur eine zentrale arbeitsökonomische und makroökonomische Kategorie. Zent Arbeitswiss 65(1):39-49

Zink KJ (2014) Arbeitswissenschaft und globales Umfeld. Zent Arbeitswiss 68(4):203-207

Zink KJ (2017) Nachhaltige Arbeitssysteme in (internationalen) Wertschöpfungsketten und die Rolle der Arbeitswissenschaft. Zent Arbeitswiss 71(2):92-100 